\title{
Assessing phytosanitary risks of growing agricultural crops in Russia and neighboring countries by a complex of phytophagous insects
}

\author{
Igor Grichanov", Elena Ovsyannikova, and Mikhail Saulich
}

All-Russian Institute of Plant Protection, Laboratory of Phytosanitary Diagnostics and Forecasts, 196608 St. Petersburg, Russia

\begin{abstract}
The relevance of the study is determined by the need to improve the monitoring of agricutural crop pest distribution. The aim of the research is to identify zones of different phytosanitary risks within the area of crop cultivation in Russia and neighboring countries. Maps characterizing the distribution and harmfulness zones of phytophagous species were used as the primary material for integrated computer analysis. It was necessary to adjust and update a number of maps taking into account research data published over the last 10 years. The analysis resulted in the creation of maps characterizing phytosanitary risk zones for the cultivation of fruit and grain crops by a corresponding complex of specialized phytophagous insects, as well as of agricultural crops in general by a complex of polyphagous pests. Three zones of phytosanitary risk have been outlined, as a consequence of the total harmfulness of phytophagous complex. The combined geoinformation system for agricultural crop pests is included in the "Phytosanitary Risks" cartographical database, which is a set of vector files for all major phytophagous species, distribution and harmfulness zone layers for these species. Outlined zones with low, medium and high phytosanitary risks can be used in the development of integrated pest management.
\end{abstract}

\section{Introduction}

Solving the problem of graphic representation of distribution and harmfulness zones for phytophagous crop pests was always a priority research task in the All-Russian Institute of Plant Protection (VIZR) since it creation. A major breakthrough in this field was achieved in 2003-2007 within the framework of the implementation of the joint project of VIZR with the All-Russian Institute of Crop Production, the Faculty of Geography and Geoecology of St. Petersburg State University, and the US Department of Agriculture (Office of International Research Programs of the Agricultural Research Service). During a relatively short period of time, using geoinformation systems, the VIZR professional team prepared

\footnotetext{
*Corresponding author: grichanov@mail.ru
} 
and published in the Internet maps for all economically significant species of arthropod phytophages, mainly insects [1]. Later, on this basis, it became possible to move to the next research stage, i.e. integrated zoning with the aim to assess the phytosanitary risks of agricultural crop growing by complexes of insect phytophages and their combined harmfulness.

\section{Materials and methods}

For automated zoning of the crop production areas according to the degree of phytosanitary risk of their cultivation, the AxioVision program built into the software of the stereomicroscope "Zeiss Discovery V12" was used, which has the function of stacking (ZStack) of several raster layers in one resulting image [2].

\section{Results and discussion}

Before the start, it was necessary to adjust and update a number of maps taking into account research data published over the last 10 years [3-5]. As an output of the operations carried out, we have automatically composed a map with zones of different integrated harmfulness of a species group of fruit crop pests (Figure 1). The combined zones of weak, medium and strong total harmfulness are highlighted. They can be considered as zones of potentially low, medium and high phytosanitary risk for fruit crop cultivation by a complex of specialized pests.

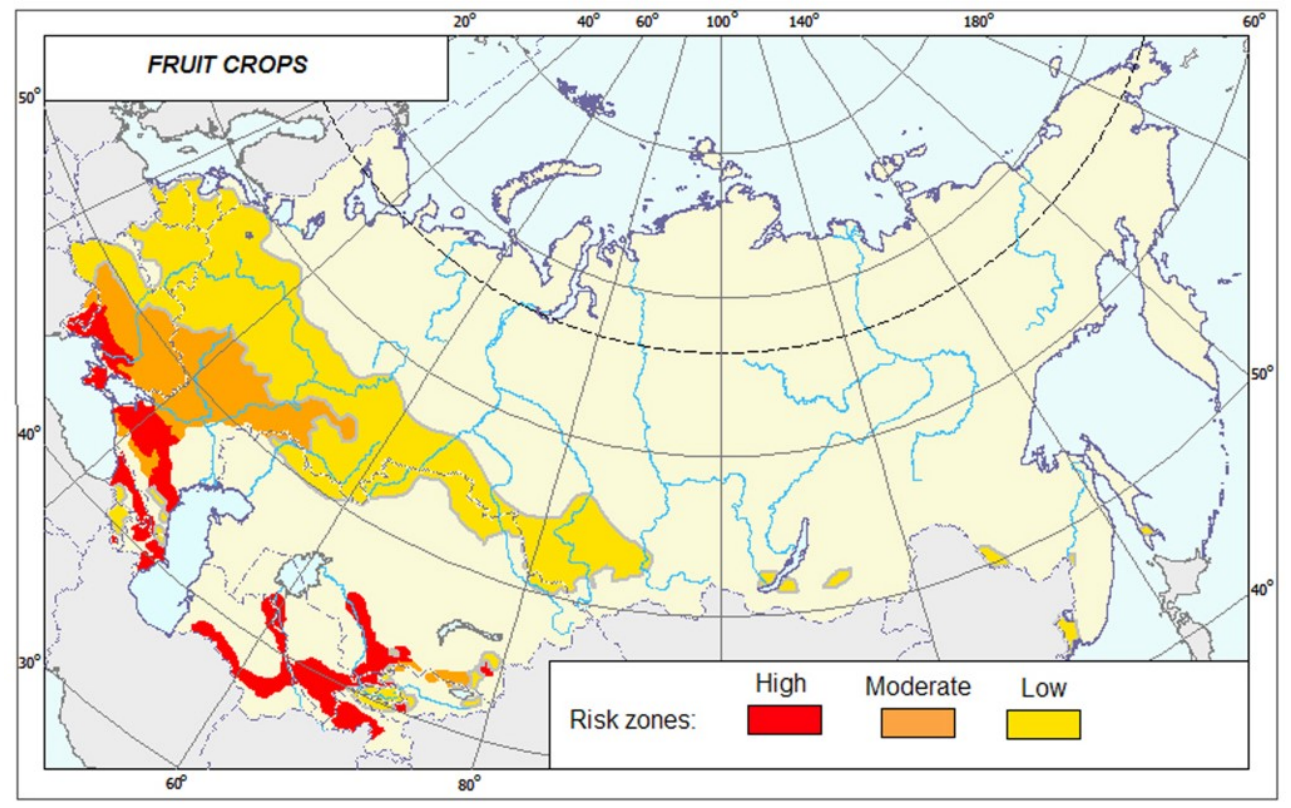

Fig. 1. Zones of potential phytosanitary risk for fruit crop cultivation by a complex of 50 specialized pest species.

The harmful fauna of grain crops is specific with regard to the degree of harmfulness of its components. Most of the species are wheat pests. Of the 28 maps of wheat pest species analyzed, seven maps have only one zone inside the area, with weak harmfulness. One map has a zone of only medium, and one more map has a zone of only strong harmfulness. 15 
maps have two harmfulness zones. Four species maps have three zones in the area, including a zone of strong harmfulness. At the same time, the share of species with a wide zone of harmfulness is relatively small. However, the economic threshold of harmfulness by yield losses is often achieved, when plants are damaged by more than one pest species, i.e. by a complex of phytophagous species, each of which causing only weak harm.

Based on the results of a comprehensive analysis of cartographic data on the harmfulness zones of 32 species of grain phytophages, the high phytosanitary risk zone is allocated, covering south-eastern Ukraine, the south of the Republic of Moldova, Crimea and the western half of the North Caucasus in the Russian Federation (Figure 2).

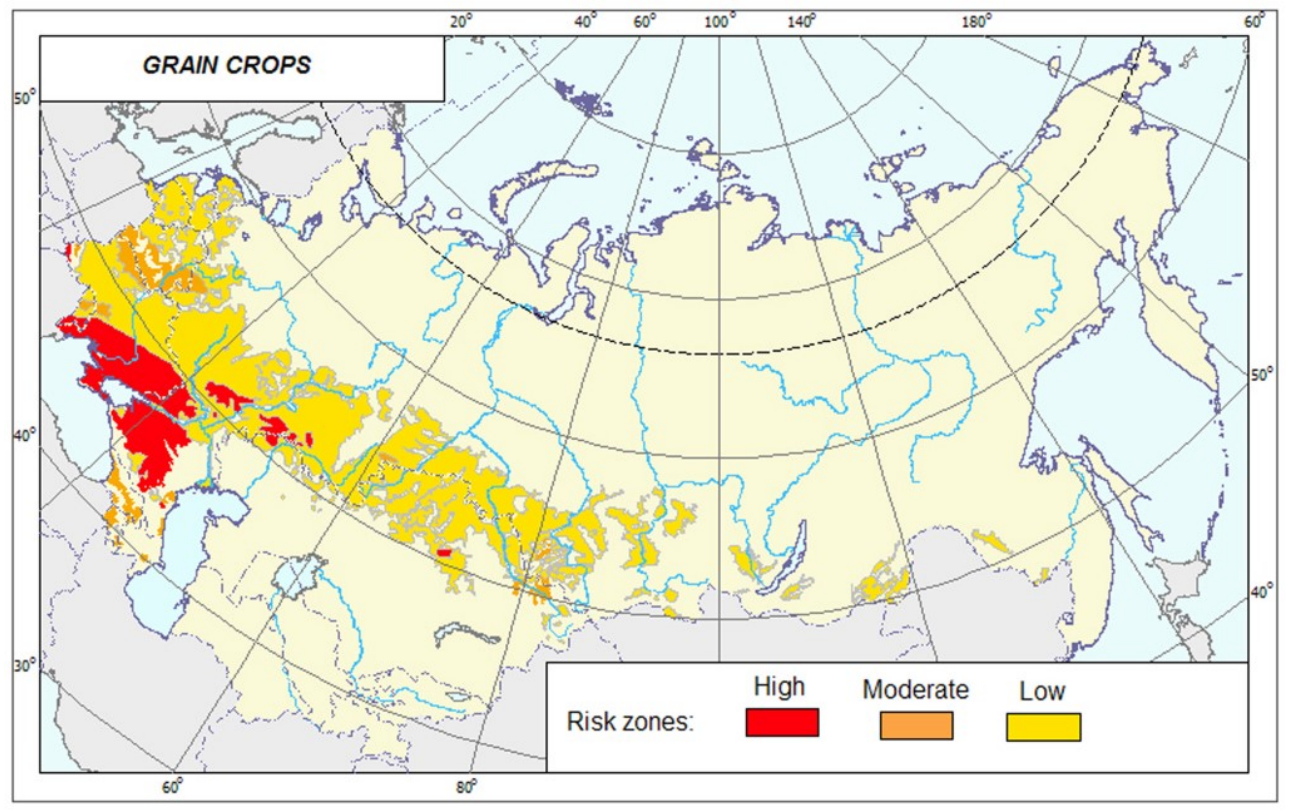

Fig. 2. Zones of potential phytosanitary risk for grain crop cultivation by a complex of 32 specialized pest species.

A comprehensive phytosanitary analysis was also carried out for a group of polyphagous pest species. The fauna of polyphagous insect phytophages has its own specificity regarding to the degree of their harmfulness. Most species belong to Lepidoptera and Coleoptera, as well as Orthoptera and Aphidoidea, with a total of 32 species. The maps for four locust species and crambid moth Loxostege sticticalis include propagation centers and only one zone of harmfulness, including two maps with strong harmfulness and three maps without a harmfulness category. One more species map has a zone of only medium, and seven species have a zone of only weak harmfulness. Ten phytophages have two zones of harmfulness, either weak and medium (8) or weak and strong (2). The maps for noctuids Silver Y (Autographa gamma) and Cotton Bollworm (Helicoverpa armigera) contains three zones including the zone of strong harmfulness. Most species have a wide zone of harmfulness and a very wide area that distinguishes polyphagous phytophages from specialized pests with predomination of only one zone of weak harmfulness. However, the economic threshold of harmfulness by yield losses of potato, sunflower, grain and other crops is often achieved, when plants are damaged by more than one pest species, i.e. by a complex of phytophagous species and specialized phytophages, each of which causing only weak harm in a certain region.

Based on the analysis of cartographic data on the harmfulness zones of 32 species of polyphagous phytophages, the high phytosanitary risk zone is highlighted, covering central 
Ukraine, Trans-Caucasian and Middle Asian valleys, the Volga and Amu Darya river mouths (Figure 3).

The combined geoinformation system for crop pests is included in the "Phytosanitary Risks" cartographic database, which is a set of vector files for all major phytophagous species, distribution and harmfulness zone layers for these species, as well as basic vector layers of "shell" [6]. We think that outlined zones with low, medium and high phytosanitary risks Outlined zones with low, medium and high phytosanitary risks can be used in the development of integrated pest management. The "Phytosanitary Risks" database, as a reference tools, can be used by researchers, students, and teachers of agricultural universities, by plant protection and quarantine service officers.

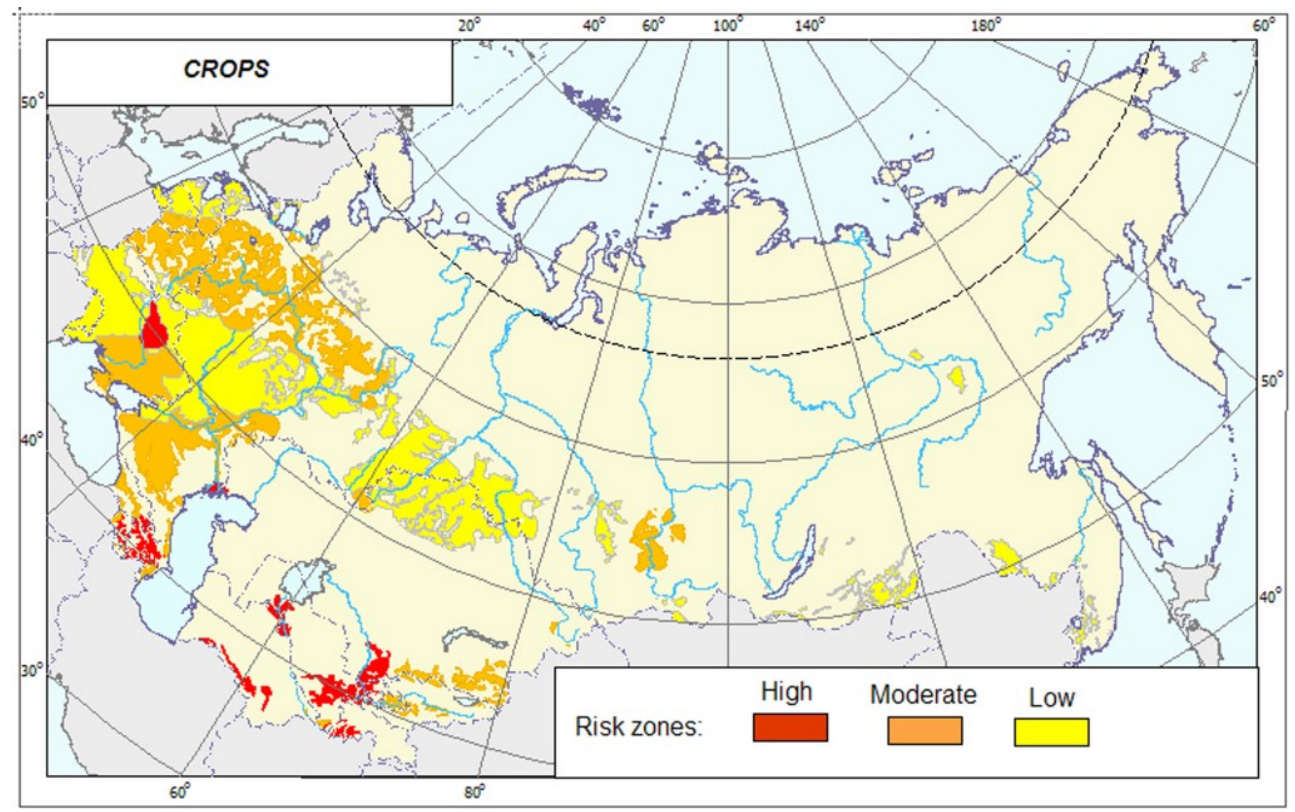

Fig. 3. Zones of potential phytosanitary risk for agricultural crop cultivation by a complex of 32 polyphagous pest species.

The work was performed within the Program for Basic Scientific Research of the Government of the Russian Federation, project \# 0665-2019-0014.

\section{References}

1. A.N. Afonin, S.L. Greene, N.I. Dzyubenko, A.N. Frolov, et al., Interactive Agricultural Ecological Atlas of Russia and Neighboring Countries. Economic Plants and their Diseases, Pests and Weeds (2008) http://www.agroatlas.ru

2. I.Ya. Grichanov, E.I. Ovsyannikova, Plodovodstvo i Vinogradarstvo Yuga Rossii, 22, 4, 1-15 (2013) http://journal.kubansad.ru/pdf/13/04/08.pdf

3. I.Ya. Grichanov, E.I. Ovsyannikova, M.I. Saulich, Maps of areas and zones of harmfulness of fruit and berry pests and diseases, 1-62 (St. Petersburg, 2016) https:/archive.org/details/GrichanovOvsyannikovaSaulich2016MapsEncrypt

4. I.Ya. Grichanov, E.I. Ovsyannikova, M.I. Saulich, Maps of areas and zones of harmfulness of grain crop pests, 1-85 (St. Petersburg, 2018) http://doi.org/10.5281/zenodo. 1257174 
5. I.Ya. Grichanov, E.I. Ovsyannikova, M.I. Saulich, Maps of areas and zones of harmfulness of polyphagous insect crop pests, 1-42 (St. Petersburg, 2019) http://doi.org/10.5281/zenodo.3461623

6. I.Ya. Grichanov, E.I. Ovsyannikova, M.I. Saulich, A.N. Frolov, V.I. Yakutkin, M.N. Berim, G.E. Davidyan, F.A. Karlik, Yu.M. Malysh, Data Base "Phytosanitary risks". Certificate on the State Registration of the Database in Rospatent No. 2017620836 (2017) https://elibrary.ru/item.asp?id=29926373 